\title{
LIMITING THEOREMS FOR GALTON-WATSON BRANCHING PROCESS
}

\author{
BY \\ Norman Levinson ${ }^{1}$
}

1. The limiting theorems for the Galton-Watson branching process have been discovered in recent years. An account of this work and references to the literature are given in Harris [3]. A discussion of the elementary aspects of the branching process is given in Feller [1, Chapter XII].

The problem for the case where extinction does not occur with probability 1 , may be formulated mathematically as follows. The probability $p_{j} \geqq 0$ that one object forms $j$ objects is given, and

$$
\sum_{0}^{\infty} p_{j}=1, \quad \sum_{j=1}^{\infty} j p_{j}=m>1 .
$$

The generating function associated with $\left\{p_{j}\right\}$ is

$$
f(s)=\sum_{0}^{\infty} p_{j} s^{j}, \quad|s| \leqq 1 .
$$

Note that $f(1)=1$ and $f^{\prime}(1)=m$. If the random variable $z_{n}$ has as its generating function $f_{n}(s)$, where

$$
f_{1}(s)=f(s), \quad f_{n+1}(s)=f\left(f_{n}(s)\right),
$$

then $f_{n}(1)=1$, and the expectation of $z_{n}$ is given by

$$
E\left(z_{n}\right)=f_{n}^{\prime}(1)=m^{n} \text {. }
$$

The variable $z_{n}$ represents the number of objects after $n$ generations. If the renormalized random variables

are considered, and if

$$
w_{n}=z_{n} / m^{n}
$$

then

$$
G_{n}(u)=\operatorname{Prob}\left(w_{n} \leqq u\right)
$$

is given for $n \geqq 0$ by

$$
\phi_{n+1}(s)=f\left(\phi_{n}(s / m)\right), \quad \mathrm{Rl} s \geqq 0,
$$

where $\phi_{0}(s)=e^{-s}$. (Note that here $-s$ is used where Harris uses $\left.s.\right)$

Note also that $\phi_{n}(0)=1$ and $\phi_{n}^{\prime}(0)=-1$, which is equivalent to

$$
\int_{0-}^{\infty} d G_{n}(u)=1, \quad \int_{0}^{\infty} u d G_{n}(u)=1
$$

Received July 17, 1958.

1 The preparation of this paper was supported by the Office of Naval Research. 
If the further assumption is made that

$$
\sum_{1}^{\infty} j^{2} p_{j}<\infty,
$$

that is, that the second moment exists, then it has been shown that

$$
\lim _{n \rightarrow \infty} \phi_{n}(s)=\phi(s),
$$

exists. Moreover $\phi^{\prime}(0)=-1$. This is equivalent to the existence of

$$
\begin{aligned}
\lim _{n \rightarrow \infty} G_{n}(u) & =G(u), \\
\int_{0-}^{\infty} d G(u) & =1 \\
\int_{0}^{\infty} u d G(u) & =1
\end{aligned}
$$

In other words, $z_{n} / m^{n}=w_{n}$ converges in distribution to the random variable $w$ with distribution function $G$, and by (1.9) $E[w]=1$. Harris [2] also showed that for $u>0, d G / d u$ exists and is continuous (except in the trivial case where $m$ is an integer and $p_{m}=1$, so that $f(s)=s^{m}$ and $\left.\phi_{n}(s)=e^{-s}\right)$.

It was remarked by Doob that for (1.6), (1.7), and (1.8) to hold, the existence of the second moment (1.5) is not necessary. However, as will be shown below, if only the first moment $m$ exists, then it is possible for (1.9) to fail, that is, for $E(w)<1$, and indeed for $E(w)=0$. That is, it is possible for $G(u) \equiv 1, u>0$. In this case then $w=0$ with probability 1 .

It is the purpose of this article to show that the existence of the second moment can be replaced by a much weaker requirement which will assure that all the limit properties mentioned above hold, including $E(w)=1$, i.e., $\phi^{\prime}(0)=-1$. Let $\gamma(t)$ be a continuous monotone nondecreasing function of $t$ for $t \geqq 1$, and let

$$
\int_{1}^{\infty} \frac{d t}{t \gamma(t)}<\infty
$$

Then the existence of the second moment can be replaced by the weaker requirement

$$
\sum_{1}^{\infty} j \gamma(j) p_{j}<\infty,
$$

and all the limit results stated above remain valid. (Examples of $\gamma(t)$ are $\left.t^{\delta},(1+\log t)^{1+\delta},(1+\log t)[\log \log (e+t)]^{1+\delta}.\right)$

It will be more convenient to assume an even less restrictive form than (1.11), namely, for some constant $C$,

$$
\sum_{j \geqq n} j p_{j} \leqq C / \gamma(n),
$$

which is obviously implied by $(1.11)$.

Further it will be shown that if

$$
\int_{1}^{\infty} \frac{d t}{t \gamma(t)}=\infty
$$


then the situation $E(w)=0$ may actually arise even though (1.12) holds. Hence the requirement (1.10) and (1.12), which is only slightly more stringent than the existence of the first moment, is indispensable for $E(w)=1$. Incidentally the existence of $d G / d u$ for $u>0$ will be demonstrated without the assumption of the existence of the second moment by a modification in the argument of Harris [2].

The case $m<1$ can also be treated by the method that follows.

2. Theorem 2.1. If $m>1$, and (1.12) and (1.10) hold, then

$$
\lim _{n \rightarrow \infty} \phi_{n}(s)=\phi(s) \text {, }
$$

$\mathrm{Rl} s \geqq 0$,

exists, where $\phi_{n}(s)$ is defined in (1.3) and

$$
\phi(s)=f(\phi(s / m)) .
$$

Moreover $\phi(0)=1$ and $\phi^{\prime}(0)=-1$ in the sense that

$$
\lim _{s \rightarrow 0}(1-\phi(s)) / s=1,
$$

$\mathrm{Rl} s \geqq 0$.

Moreover $(2.2), \phi(0)=1$, and (2.3) determine $\phi(s)$ uniquely even if $s$ is replaced by the real variable $\sigma$.

This theorem will be proved by means of the following lemmas.

Lemma 2.1. The formulas (1.12) and (1.10) imply the existence of a continuous nondecreasing function $\alpha(\sigma), 0 \leqq \sigma<\infty, \alpha(0)=0$, such that

$$
\begin{gathered}
\int_{0}^{1} \frac{\alpha(\sigma)}{\sigma} d \sigma<\infty, \\
\left|\left(1-f\left(e^{-\sigma}\right)\right) / \sigma-m\right| \leqq \alpha(\sigma), \quad 0 \leqq \sigma<\infty .
\end{gathered}
$$

Proof of Lemma 2.1. It is convenient to introduce $\beta(t)=C / \gamma(t)$. Since (1.10) implies $\gamma(\infty)=\infty, \beta(\infty)=0$. Also $\beta(t)$ is continuous and nonincreasing for $1 \leqq t<\infty$, and by (1.10) and (1.12)

$$
\int_{1}^{\infty} \frac{\beta(t)}{t} d t<\infty,
$$

and

$$
\sum_{j \geqq n} j p_{j} \leqq \beta(n) .
$$

With no restriction it can be assumed that $\beta$ has a continuous derivative; indeed, replace it by $\int_{t-1}^{t} \beta(y) d y$.

From the definition of $m$ and $f$ follows for $\sigma>0$

since

$$
m-\left(1-f\left(e^{-\sigma}\right)\right) / \sigma=\sum_{j=1}^{\infty} j p_{j}\left(1-\left(1-e^{-j \sigma}\right) / j \sigma\right)
$$

$$
\begin{aligned}
& 0<1-\left(1-e^{-x}\right) / x<\min (x, 1) \quad \text { for } 0<x<\infty, \\
& \left|m-\left(1-f\left(e^{-\sigma}\right)\right) / \sigma\right| \leqq J_{1}+J_{2}
\end{aligned}
$$


for $0<\sigma<1$, where

$$
J_{1}=\sum_{j \geqq 1 / \sigma} j p_{j} \leqq \beta(1 / \sigma)
$$

and

If $S_{j}=\sum_{k \geqq j} k p_{k}$, then

$$
J_{2}=\sigma \sum_{j<1 / \sigma} j^{2} p_{j}
$$

$$
\begin{array}{r}
J_{2}=\sigma \sum_{j<1 / \sigma} j\left(S_{j}-S_{j+1}\right)=\sigma \sum_{j<1 / \sigma} j S_{j}-\sigma \sum_{j-1<1 / \sigma}(j-1) S_{j} \\
\leqq \sigma \sum_{j<1 / \sigma} S_{j} \leqq \sigma \sum_{j<1 / \sigma} \beta(j)<\sigma \beta(1)+\sigma \int_{1}^{1 / \sigma} \beta(t) d t .
\end{array}
$$

For $0 \leqq \sigma \leqq 1$, let

$$
\alpha(\sigma)=\beta(1 / \sigma)+\sigma \beta(1)+\sigma \int_{1}^{1 / \sigma} \beta(t) d t,
$$

and let $\alpha(\sigma)=\alpha(1)$ for $\sigma>1$. Then by (2.8), (2.9), and (2.10), it follows that (2.5) holds. Moreover $\alpha$ is continuous, and

$$
\alpha^{\prime}(\sigma)=-\frac{1}{\sigma^{2}} \beta^{\prime}(1 / \sigma)+\beta(1)+\int_{1}^{1 / \sigma} \beta(t) d t-\frac{1}{\sigma} \beta(1 / \sigma) .
$$

Since $\beta^{\prime} \leqq 0$,

$$
\int_{1}^{1 / \sigma} \beta(t) d t \geqq\left(\frac{1}{\sigma}-1\right) \beta(1 / \sigma),
$$

and $\beta(1) \geqq \beta(1 / \sigma)$. It follows that $\alpha^{\prime}(\sigma) \geqq 0$. Hence $\alpha$ is nondecreasing, and $\alpha(0)=0$. Moreover,

$$
\int_{0}^{1} \frac{\alpha(\sigma)}{\sigma} d \sigma=\int_{0}^{1} \frac{\beta(1 / \sigma)}{\sigma} d \sigma+\beta(1)+\int_{0}^{1}\left(\int_{1}^{1 / \sigma} \beta(t) d t\right) d \sigma .
$$

Setting $\sigma=1 / u$ gives

$$
\begin{gathered}
\int_{0}^{1} \frac{\beta(1 / \sigma) d \sigma}{\sigma}=\int_{1}^{\infty} \frac{\beta(u)}{u} d u \\
\int_{0}^{1}\left(\int_{1}^{1 / \sigma} \beta(t) d t\right) d \sigma=\int_{1}^{\infty}\left(\int_{1}^{u} \beta(t) d t\right) \frac{d u}{u^{2}}=\int_{1}^{\infty} \frac{\beta(t)}{t} d t .
\end{gathered}
$$

Hence (2.4) holds, and the lemma is proved.

Lemma 2.2. Let (2.4) and (2.5) hold. Let $\phi_{0}(\sigma)=e^{-\sigma}$, and let

$$
\phi_{n+1}(\sigma)=f\left(\phi_{n}(\sigma / m)\right), \quad 0 \leqq \sigma<\infty .
$$

Then over any finite interval of $0 \leqq \sigma<\infty, \phi_{n}(\sigma)$ converges uniformly to a limit $\phi(\sigma)$. Moreover $\phi(0)=1$,

$$
\phi(\sigma)=f(\phi(\sigma / m)),
$$

and

$$
\lim _{\sigma \rightarrow 0+}(1-\phi(\sigma)) / \sigma=1 .
$$


Proof of Lemma 2.2. By induction, $0 \leqq \phi_{n}(\sigma) \leqq 1$. Since $\left|f^{\prime}(s)\right| \leqq m$ for $|s| \leqq 1$,

$$
\phi_{n+1}(\sigma)-\phi_{n}(\sigma)=f\left(\phi_{n}(\sigma / m)\right)-f\left(\phi_{n-1}(\sigma / m)\right)
$$

yields by the theorem of the mean

$$
\left|\phi_{n+1}(\sigma)-\phi_{n}(\sigma)\right| \leqq m\left|\phi_{n}(\sigma / m)-\phi_{n-1}(\sigma / m)\right|
$$

Clearly

$\phi_{1}(\sigma)-\phi_{0}(\sigma)=f\left(e^{-\sigma / m}\right)-e^{-\sigma}=\frac{\sigma}{m}\left[m-\frac{1-f\left(e^{-\sigma / m}\right)}{\sigma / m}\right]+1-\sigma-e^{-\sigma}$.

Since $\left|1-\sigma-e^{-\sigma}\right| \leqq \sigma^{2}$,

$$
\left|\phi_{1}(\sigma)-\phi_{0}(\sigma)\right| \leqq(\sigma / m) \alpha(\sigma / m)+\sigma^{2} .
$$

If $\tilde{\alpha}(\sigma)=\alpha(\sigma)+m^{2} \sigma$, then $\tilde{\alpha}$ has all the properties of $\alpha$, and

$$
\left|\phi_{1}(\sigma)-\phi_{0}(\sigma)\right| \leqq(\sigma / m) \tilde{\alpha}(\sigma / m) \text {. }
$$

Hence denoting $\tilde{\alpha}$ from here on by $\alpha$, we have

$$
\left|\phi_{1}(\sigma)-\phi_{0}(\sigma)\right| \leqq(\sigma / m) \alpha(\sigma / m) .
$$

Using (2.14) and (2.15) yields

Hence for $k>0$

$$
\left|\phi_{n+1}(\sigma)-\phi_{n}(\sigma)\right| \leqq(\sigma / m) \alpha\left(\sigma / m^{n+1}\right) .
$$

$$
\left|\phi_{n+k}(\sigma)-\phi_{n}(\sigma)\right| \leqq(\sigma / m) \sum_{j=1}^{\infty} \alpha\left(\sigma / m^{n+j}\right) .
$$

Since $\alpha$ is nondecreasing and $m>1$,

Thus

$$
\alpha\left(\sigma / m^{n+j}\right) \leqq \alpha\left(\sigma / m^{n+t}\right), \quad j-1 \leqq t \leqq j .
$$

$$
\sum_{j=1}^{\infty} \alpha\left(\sigma / m^{n+j}\right) \leqq \int_{0}^{\infty} \alpha\left(\sigma / m^{n+t}\right) d t
$$

Let $t$ be replaced by the variable $u$, where

Thus

$$
u=\sigma / m^{n+t}, \quad d t=-d u /(u \log m) .
$$

Hence

$$
\int_{0}^{\infty} \alpha\left(\sigma / m^{n+t}\right) d t=\frac{1}{\log m} \int_{0}^{\sigma / m^{n}} \frac{\alpha(u)}{u} d u .
$$

$$
\left|\phi_{n+k}(\sigma)-\phi_{n}(\sigma)\right| \leqq \frac{\sigma}{m \log m} \int_{0}^{\sigma / m^{n}} \frac{\alpha(u)}{u} d u .
$$

This proves the uniform convergence of $\phi_{n}(\sigma)$ over any finite interval of $\sigma$ as $n \rightarrow \infty$, and (2.12) follows from (2.11). Since $\phi_{n}(0)=1$, it follows that $\phi(0)=1$.

From (2.16) follows with $n=0$

$$
\left|\frac{1-\phi_{k}(\sigma)}{\sigma}-\frac{1-\phi_{0}(\sigma)}{\sigma}\right| \leqq \frac{1}{m \log m} \int_{0}^{\sigma} \frac{\alpha(u)}{u} d u .
$$


Letting $k \rightarrow \infty$ gives

$$
\left|\frac{1-\phi(\sigma)}{\sigma}-\frac{1-e^{-\sigma}}{\sigma}\right| \leqq \frac{1}{m \log m} \int_{0}^{\sigma} \frac{\alpha(u)}{u} d u .
$$

Now letting $\sigma \rightarrow 0+$ gives (2.13).

Proof of Theorem 2.1. With $\phi_{n}(s)$ defined in terms of $f$ by the recursion formula (1.3) and $\phi_{0}(s)=e^{-s}$, it follows by induction that

$$
\phi_{n}(s)=\sum_{j=0}^{\infty} p_{n, j} e^{-j s / m^{n}},
$$

where the $p_{n, j} \geqq 0$. Since $\phi_{n}(0)=1, \phi_{n}^{\prime}(0)=-1$,

$$
\sum_{j=0}^{\infty} p_{n, j}=1, \quad \sum_{j=1}^{\infty} j p_{n, j}=1 .
$$

Hence $\phi_{n}(s), \mathrm{Rl} s \geqq 0$, is the Laplace-Stieltjes transform of a step function $G_{n}(u)$ which increases at $u=j / m^{n}$ by $p_{n, j}$. Thus $G_{n}(u)=0, u<0$, and $G_{n}(\infty)=1$, and

$$
\phi_{n}(s)=\int_{0-}^{\infty} e^{-s u} d G_{n}(u), \quad \text { Rl } s \geqq 0 .
$$

By the Helly selection theorem it would follow, if the $G_{n}(u)$ did not converge as $n \rightarrow \infty$, that there would be at least two subsequences each converging to distinct limits, $G$ and $\tilde{G}$. These would have distinct transforms, $\phi(s)$ and $\tilde{\phi}(s)$. But by Lemma 2.2, $\phi(\sigma)-\tilde{\phi}(\sigma) \equiv 0$. Since $\phi$ and $\tilde{\phi}$ are analytic for Rl $s>0$, this implies that $\phi \equiv \tilde{\phi}$, and hence that $G_{n}(u)$ must converge to a limit $G(u)$. Hence by $(2.17), \phi_{n}(s)$ converges to $\phi(s)$ for $\mathrm{Rl} s \geqq 0$.

From (2.13) and

follows readily that

$$
\phi(\sigma)=\int_{0-}^{\infty} e^{-\sigma u} d G(u)
$$

$$
\int_{0-}^{\infty} u d G(u)=1
$$

and this in turn yields (2.3). The equation (2.2) follows from (1.3) and the convergence of $\boldsymbol{\phi}_{n}(s)$.

To prove that $\phi(\sigma)$ is uniquely determined by $(2.12),(2.13)$, and $\phi(0)=1$, observe that (2.13) implies

$$
\phi(\sigma)=1-\sigma+\sigma o(1)
$$

for small $\sigma>0$. Let $\tilde{\phi}(\sigma)$ also satisfy these conditions. Then if

$$
|\tilde{\phi}(\sigma)-\phi(\sigma)|=\sigma \tau(\sigma),
$$

$\tau(\sigma) \geqq 0$, and $\tau(\sigma) \rightarrow 0$ as $\sigma \rightarrow 0+$. From

$$
\tilde{\phi}(m \sigma)-\phi(m \sigma)=f(\tilde{\phi}(\sigma))-f(\phi(\sigma))
$$

and $\left|f^{\prime}\right| \leqq m$ follows

$$
|\tilde{\phi}(m \sigma)-\phi(m \sigma)| \leqq m|\tilde{\phi}(\sigma)-\phi(\sigma)|,
$$


or

$$
\tau(m \sigma) \leqq \tau(\sigma),
$$$$
\sigma \geqq 0 \text {. }
$$

Since $\tau(\sigma) \rightarrow 0$ as $\sigma \rightarrow 0+$ and $m>1,(2.18)$ implies that $\tau(\sigma) \equiv 0$, which proves that $(2.12),(2.13)$, and $\phi(0)=1$ determine $\phi(\sigma)$ uniquely.

3. It will be shown here that if the conditions (1.10) and (1.12) are weakened, then the case $\phi(s) \equiv 1$ can arise, that is, $E(w)=0$. It will be more convenient to work with $\beta(t)=C / \gamma(t)$ as was done in the proof of Lemma 2.1. Let $\beta(t)$ be continuous and decreasing for $t \geqq 1$. Let

$$
\int_{1}^{\infty} \frac{\beta(t)}{t} d t=\infty
$$

Further let $\beta^{\prime}(t)$ exist, $\beta^{\prime}(t)<0$, and let $\beta^{\prime}(t)$ be increasing. (Examples of such $\beta(t)$ are $[\log (1+t)]^{-1},[\log (e+t) \log \log (e+t)]^{-1}$, etc. $)$ Then it will be shown that there exist $p_{j} \geqq 0, j \geqq 0$, and a constant $v>0$ such that

$$
\begin{gathered}
\sum_{0}^{\infty} p_{j}=1, \quad \sum_{0}^{\infty} j p_{j}=m>1, \\
\sum_{j \geqq n} j p_{j} \leqq v \beta(n),
\end{gathered}
$$

and such that $\phi_{n}(s) \rightarrow 1$ as $n \rightarrow \infty, \mathrm{Rl} s \geqq 0$.

Lemma 3.1. With $\beta(t)$ as described above satisfying (3.1), there exist $\left\{p_{j}\right\}$ satisfying (3.2) and (3.3) and a continuous increasing function $b(\sigma), 0 \leqq \sigma \leqq 1$, $b(0)=0$, such that

$$
m-(1-f(\sigma)) /(1-\sigma) \geqq b(1-\sigma), \quad 0 \leqq \sigma \leqq 1,
$$

and

$$
\int_{0}^{1} \frac{b(\sigma)}{\sigma} d \sigma=\infty
$$

where as usual $f(s)=\sum p_{j} s^{j}$.

Proof of Lemma 3.1. Let $p_{0}=p_{1}=0$, and let

$$
p_{j}=-\left(v \beta^{\prime}(j+1)\right) / j
$$

where $v$ is chosen so that $\sum p_{j}=1$. That this can be done follows from

$$
\sum_{j=n}^{\infty} j p_{j}=-v \sum_{j=n}^{\infty} \beta^{\prime}(j+1) \leqq-v \int_{n}^{\infty} \beta^{\prime}(t) d t=v \beta(n),
$$

which proves (3.3). Clearly 
Let

$$
\begin{aligned}
m-\frac{1-f(\sigma)}{1-\sigma} & =-v \sum_{2}^{\infty} \beta^{\prime}(j+1)\left[1-\frac{1-\sigma^{j}}{(1-\sigma) j}\right] \\
& \geqq-v \sum_{j>2 /(1-\sigma)} \beta^{\prime}(j+1)\left[1-\frac{1-\sigma^{j}}{(1-\sigma) j}\right] \\
& \geqq-\frac{1}{2} v \sum_{j>2 /(1-\sigma)} \beta^{\prime}(j+1) \\
& \geqq-\frac{1}{2} v \int_{2 /(1-\sigma)}^{\infty} \beta^{\prime}(3+t) d t \\
& =\frac{1}{2} v \beta\left(3+\frac{2}{1-\sigma}\right) .
\end{aligned}
$$

$$
b(\sigma)=\frac{1}{2} v \beta(3+2 / \sigma),
$$$$
0 \leqq \sigma \leqq 1 .
$$

Then the above is (3.4), and by letting $\sigma=1 / t$,

$$
\int_{0}^{1} \frac{b(\sigma)}{\sigma} d \sigma=\frac{1}{2} v \int_{0}^{1} \beta(3+2 / \sigma) \frac{d \sigma}{\sigma}=\frac{1}{2} v \int_{1}^{\infty} \beta(3+2 t) \frac{d t}{t}=\infty
$$

by (3.1). Hence (3.5) is proved.

By letting $\phi_{0}(\sigma)=e^{-\sigma}$ and, as in (1.3),

$$
\phi_{n+1}(\sigma)=f\left(\phi_{n}(\sigma / m)\right),
$$

it follows from the theory of martingales, as Doob has pointed out, that $\phi_{n}(\sigma)$ converges to a limit $\phi(\sigma)$ for $\sigma \geqq 0$. Since $f^{\prime}$ and $f^{\prime \prime}>0$, an easy induction shows that $\phi_{n}^{\prime \prime}(\sigma)>0$ for $0<\sigma<\infty$. Let

$$
\lambda_{n}(\sigma)=\left(1-\phi_{n}(\sigma)\right) / \sigma
$$$$
0<\sigma<\infty .
$$

Then $\lambda_{n}(0)=1$ since $-\phi_{n}^{\prime}(0)=1$. Also

Because $\phi_{n}^{\prime \prime}>0$,

$$
\lambda_{n}^{\prime}(\sigma)=-\left(1-\phi_{n}(\sigma)+\sigma \phi_{n}^{\prime}(\sigma)\right) / \sigma^{2} .
$$

$$
\left(1-\phi_{n}(\sigma)\right) / \sigma>-\phi_{n}^{\prime}(\sigma) .
$$

Hence $\lambda_{n}^{\prime}(\sigma)<0$, and so $\lambda_{n}(\sigma)$ is a decreasing function. Since $\phi_{n}(\sigma)<1$, for $\sigma>0, \lambda_{n}(\sigma)>0$. Since $\phi_{n}^{\prime}(0)=-1$, it follows that

$$
\lim _{\sigma \rightarrow 0+}(1-\phi(\sigma)) / \sigma=\int_{0}^{\infty} u d G(u) \leqq 1 .
$$

Assume that

$$
\lim _{\sigma \rightarrow 0+}(1-\phi(\sigma)) / \sigma=c>0 .
$$

Then there exists $\sigma_{1}>0$ such that

$$
\left(1-\phi\left(\sigma_{1}\right)\right) / \sigma_{1}>\frac{2}{3} c .
$$


Since $\phi_{n}(\sigma) \rightarrow \phi(\sigma)$, there exists $n_{0}>0$ such that

$$
\left(1-\phi_{n}\left(\sigma_{1}\right)\right) / \sigma_{1}>\frac{1}{2} c,
$$

or $\lambda_{n}\left(\sigma_{1}\right) \geqq c / 2$ for $n \geqq n_{0}$. Since $\lambda_{n}(\sigma)$ is decreasing

$$
\lambda_{n}(\sigma) \geqq \frac{1}{2} c, \quad n \geqq n_{0}, \quad 0 \leqq \sigma \leqq \sigma_{1} .
$$

If the function $A(\sigma)$ is defined by

$$
f(\sigma)=1+(\sigma-1) m+(1-\sigma) A(1-\sigma), \quad 0<\sigma<1,
$$

then because $f^{\prime \prime}>0, A(\sigma)>0,0 \leqq \sigma<1$. From (3.6) and (3.9) follows

$$
\begin{aligned}
& \lambda_{n+1}(\sigma)=\frac{1-f\left(\phi_{n}(\sigma / m)\right)}{\sigma} \\
&=\frac{m}{\sigma}\left[1-\phi_{n}(\sigma / m)\right]-\frac{1-\phi_{n}(\sigma / m)}{\sigma} A\left[1-\phi_{n}(\sigma / m)\right]
\end{aligned}
$$

or

$$
\lambda_{n+1}(\sigma)=\lambda_{n}(\sigma / m)\left[1-m^{-1} A\left((\sigma / m) \lambda_{n}(\sigma / m)\right)\right] .
$$

By (3.4), $A(\sigma) \geqq b(\sigma)$. Hence

$$
\lambda_{n+1}(\sigma) \leqq \lambda_{n}(\sigma / m)\left[1-m^{-1} b\left((\sigma / m) \lambda_{n}(\sigma / m)\right)\right] .
$$

Since $b$ is increasing, it follows from (3.8) that if $\sigma \leqq \sigma_{1}$ and $n \geqq n_{0}$,

Hence

$$
\lambda_{n+1}(\sigma) \leqq \lambda_{n}(\sigma / m)\left[1-m^{-1} b(c \sigma / 2 m)\right] \text {. }
$$

$$
\lambda_{n+1}(\sigma) \leqq \lambda_{n}(\sigma / m) \exp \left[-m^{-1} b(c \sigma / 2 m)\right] .
$$

For $n-1$ in place of $n$ and $\sigma / m$ in place of $\sigma$,

$$
\lambda_{n}(\sigma / m) \leqq \lambda_{n-1}\left(\sigma / m^{2}\right) \exp \left[-m^{-1} b\left(c \sigma / 2 m^{2}\right)\right] \text {, etc. }
$$

Hence for $0 \leqq \sigma \leqq \sigma_{1}$

$$
\lambda_{n+1}(\sigma) \leqq \lambda_{n_{0}}\left(\sigma / m^{n+1-n_{0}}\right) \exp \left[-m^{-1} \sum_{k=0}^{n-n_{0}} b\left(c \sigma /\left(2 m^{k+1}\right)\right)\right] .
$$

Since $b$ is increasing,

$$
\sum_{k=0}^{n-n_{0}} b\left(c \sigma /\left(2 m^{k+1}\right)\right) \geqq \int_{0}^{n-n_{0}} b\left(c \sigma /\left(2 m^{t+1}\right)\right) d t .
$$

Let $u=c \sigma /\left(2 m^{t+1}\right)$. Then

and

$$
t+1=\frac{1}{\log m} \log (c \sigma / 2 u)
$$

$$
\int_{0}^{n-n_{0}} b\left(c \sigma /\left(2 m^{t+1}\right)\right) d t=\int_{c \sigma /\left(2 m^{\left.n-n_{0}+1\right)}\right.}^{c \sigma /(2 m)} \frac{b(u)}{u} d u / \log m .
$$

If we let $n \rightarrow \infty$, this integral diverges by (3.5). Since $\lambda_{n_{0}}(\sigma) \leqq 1$, letting $n \rightarrow \infty$ in (3.10) gives

$$
\lim _{n \rightarrow \infty} \lambda_{n}(\sigma)=0, \quad 0<\sigma \leqq \sigma_{1},
$$


which contradicts (3.8) and hence (3.7). Thus $c=0$ in (3.7), and this implies

$$
\int_{0}^{\infty} u d G(u)=0
$$

which in turn implies that $\phi(s) \equiv 1$ and $E(w)=0$.

4. To prove that $G(u)$, the limiting distribution of $w=\lim _{n \rightarrow \infty} w_{n}$, has a continuous derivative for $u>0$, and further that $G(0+)=a$, where $a$ is the least nonnegative solution of $f(\sigma)=\sigma$, the procedure of Harris [2] can be used with one modification, which will now be discussed. More precisely it will be shown $G^{\prime}(u)$ is continuous for $u>0$ except in the trivial case when $p_{j}=0$ for all $j$ except one. In this case $m$ must be an integer, and $p_{m}=1$. It follows trivially that $\phi_{n}(s)=e^{-s}$ for all $n$, and hence here $G(u)$ is a step function with a unit jump at $u=1$.

The only place where Harris [2] makes use of the existence of the second moment is to prove that $|\phi(s)|$ cannot be 1 near $s=0$ for $\mathrm{Rl} s \geqq 0$ except at $s=0$ itself. It will be shown that this follows from the existence of the first moment. Actually this property is needed, not for $\phi(s)$, but for a closely related function $\psi(s)$ which will now be defined.

With $a$ defined as the least nonnegative solution of $f(\sigma)=\sigma$, it follows since $m>1$ that $a<1$. Let

$$
k(s)=(f[s(1-a)+a]-a) /(1-a) .
$$

Then it is clear that $k$ is a probability generating function and $k(0)=0$, $k(1)=1$, and $k^{\prime}(1)=m$. (If $f(0)=0$, then $k=f$.) Moreover

$$
k(s)=\sum_{1}^{\infty} q_{j} s^{j} .
$$

Lemмa 4.1. There exists a function $\alpha_{1}(\sigma)$, with the same properties as $\alpha(\sigma)$ of Lemma 2.1, which is related to $k(\sigma)$ in the same way as $\alpha(\sigma)$ is related to $f(\sigma)$.

The straightforward proof will be given at the end of the section.

From this lemma it follows as in $\$ 2$ for $f(s)$, that there exists a unique $\psi(s)$ or $\mathrm{Rl} s \geqq 0$ such that

$$
\begin{gathered}
\psi(m s)=k(\psi(s)), \\
\psi(0)=1, \quad \lim _{\sigma \rightarrow 0+}(1-\psi(\sigma)) / \sigma=1,
\end{gathered}
$$

and $\psi(s)$ is given by

$$
\psi(s)=\int_{0-}^{\infty} e^{-s u} d H(u)
$$

where $H(u)$ is related to $\psi$ as $G$ is to $\phi$ in $\$ 2$. $\quad H(u)$ is a distribution function. Since (4.2) and (4.3) determine $\psi$ uniquely, direct verification shows that

$$
\psi(s)=(\phi[(1-a) s]-a) /(1-a) .
$$


Recalling that $\phi$ is the Laplace-Stieltjes transform of $G$, it follows from (4.5) that

$$
\begin{aligned}
& H(u)=\frac{G(u /(1-a))-a}{1-a}, \quad u \geqq 0, \\
& H(u)=0, \quad u<0 .
\end{aligned}
$$

To show that $G(0+)=a$ and that $G^{\prime}(u)$ is continuous for $u>0$, it suffices to show that $H(u)$ is continuous for $-\infty<u<\infty$, and that $H^{\prime}(u)$ is continuous for $u>0$. The proof of this given by Harris is valid once it is shown that $|\psi(s)|$ is less than 1 for $s \neq 0, \mathrm{Rl} s \geqq 0$, and $|s|$ small. Since $H(u)$ is a distribution function with first moment 1 , it follows from (4.4) that, if $s=\sigma+i t$

$$
|\psi(\sigma+i t)|<1, \quad \sigma>0 .
$$

Hence if $|\psi(s)|$ assumes the value 1 arbitrarily close to $s=0$, it must be at a sequence of points $i t_{j}, t_{j} \rightarrow 0$. It will be assumed in the following argument that $t_{j}>0$. The case $t_{j}<0$ can be dealt with in a similar way, and the general case can be reduced to the one or the other by discarding some $t_{j}$. If $\left|\psi\left(i t_{j}\right)\right|=1$, then

$$
\int_{0-}^{\infty} e^{-i t_{j} u} d H(u)=e^{-i c_{j}},
$$

where $c_{j}$ is real and $0 \leqq c_{j}<2 \pi$. From (4.7)

$$
\int_{0-}^{\infty} e^{-i\left(t_{j} u-c_{j}\right)} d H(u)=1 .
$$

Since $H(u)$ is a distribution function, (4.8) implies that $H(u)$ is a step function which can increase only for those $u$ for which

$$
t_{j} u=c_{j}+2 n \pi,
$$

where $n \geqq 0$ is an integer. Since $t_{j} \rightarrow 0$, it follows that for large $j, H(u)$ can have a jump at $c_{j} / t_{j}$ and nowhere else. Thus $c_{j} / t_{j}$ is a constant. Since $H(u)$ is a step function with only one jump and first moment 1 , the jump must be at $u=1$, and hence $\psi(s)=e^{-s}$.

From $\psi(m s)=k(\psi(s))$ and the series representation of $k$,

$$
e^{-m s}=\sum_{1}^{\infty} q_{j} e^{-j s} .
$$

Hence $m$ must be an integer, $q_{m}=1$, and all other $q_{j}=0$. Thus $k(s)=s^{m}$. From (4.1)

$$
f[s(1-a)+a]=a+(1-a) s^{m} .
$$

Differentiating and setting $s=0$ gives $f^{\prime}(a)=0$. This implies $a=0$, and hence $f(s)=s^{m}$, which was ruled out in our hypothesis concerning $f$. This completes the proof that $|\psi(s)|<1, s \neq 0,|s|$ small, and Rl $s \geqq 0$.

Proof of Lemma 4.1. Let

$$
f\left(e^{-\sigma}\right)=1-\sigma m+\sigma r(\sigma) .
$$


Then by (2.5)

$$
|r(\sigma)| \leqq \alpha(\sigma)
$$

Thus $r(\sigma)=o(1)$ as $\sigma \rightarrow 0+$. From (4.1) and (4.9)

$$
\begin{aligned}
(1-a) k\left(e^{-\sigma}\right) & =(1-a)+m \log \left[1-(1-a)\left(1-e^{-\sigma}\right)\right] \\
& -\log \left[1-(1-a)\left(1-e^{-\sigma}\right)\right] r\left[\log \frac{1}{1-(1-a)\left(1-e^{-\sigma}\right)}\right] .
\end{aligned}
$$

For small $x, \log (1-x)=-x+O\left(x^{2}\right)$. Hence

(4.11) $k\left(e^{-\sigma}\right)=1-m \sigma+\sigma r_{1}(\sigma)+\sigma r\left[\log \frac{1}{1-(1-a)\left(1-e^{-\sigma}\right)}\right]$,

where $r_{1}(\sigma)=O(\sigma)$ for small $\sigma$. Let

Then $\rho(0)=0$, and

$$
\rho(\sigma)=\frac{1}{1-a} \log \frac{1}{1-(1-a)\left(1-e^{-\sigma}\right)} .
$$

$$
\rho^{\prime}(\sigma)=1 /\left((1-a)+a e^{\sigma}\right)
$$

so that $\rho^{\prime}(\sigma)>0$, and $\rho^{\prime}(0)=1$. Hence by (4.10)

$$
\left|r\left[\log \frac{1}{1-(1-a)\left(1-e^{-\sigma}\right)}\right]\right| \leqq \alpha[(1-a) \rho(\sigma)] .
$$

If $(1-a) \rho(\sigma)=t$ inverts to give $\sigma=\Gamma(t)$, then $\Gamma(0)=0, \Gamma^{\prime}(t)>0$, and $\Gamma^{\prime}(0)=1 /(1-a)$. Hence

$$
\int_{0}^{1} \alpha[(1-a) \rho(\sigma)] \frac{d \sigma}{\sigma}=\int_{0}^{(1-a) \rho(1)} \frac{\alpha(t)}{t}\left[\frac{t \Gamma^{\prime}(t)}{\Gamma(t)}\right] d t ;
$$

since the bracketed term tends to 1 as $t \rightarrow 0$, the integral converges. Thus if $A>1$ is an appropriate constant, and if

$$
\alpha_{1}(\sigma)=\alpha[(1-a) \rho(\sigma)]+A \sigma,
$$

$0 \leqq \sigma \leqq 1$

and $\alpha_{1}(\sigma)=\alpha_{1}(1)$ for $\sigma>1$, then by (4.11) and (4.12)

$$
0 \leqq m-\left(1-k\left(e^{-\sigma}\right)\right) / \sigma \leqq \alpha_{1}(\sigma),
$$

and $\alpha_{1}(\sigma)$ satisfies the same requirement as $\alpha(\sigma)$ in Lemma 2.1. This proves Lemma 4.1.

\section{REFERENCES}

1. William Feller, An introduction to probability theory and its applications, vol. 1, $2^{\text {nd }}$ ed., New York, 1957.

2. T. E. HARRIS, Branching processes, Ann. Math. Statist., vol. 19 (1948), pp. 474-494.

3. - - Some mathematical models for branching processes, Proceedings of the Second Berkeley Symposium on Mathematical Statistics and Probability, Berkeley, 1951, pp. 305-328.

Massachusetts Institute of Technology

Cambridge, Massachusetts 